\title{
Single- and Multi-Whisker Channels in the Ascending Projections from the Principal Trigeminal Nucleus in the Rat
}

\author{
Pierre Veinante and Martin Deschênes \\ Centre de Recherche Université Laval-Robert Giffard, Hôpital Robert Giffard, Québec G1J 2G3, Canada
}

This study investigated the relationship between axonal projections and receptive field properties of whisker-sensitive cells in the principal trigeminal sensory nucleus of the rat. The labeling of small groups of trigeminothalamic axons with biotinylated dextran amine disclosed two broad classes of axons; a majority of fibers $(68 \% ; n=107)$ project to a single barreloid of the ventral posteromedial nucleus, and the remaining group includes axons that innervate both the posterior group of the thalamus and the tectum. Additional terminal sites for axons of this latter group may include the pretectum, the zona incerta, the medial part of the medial geniculate nucleus, and the ventral posteromedial nucleus. Corresponding to these two classes of fibers, $67 \%$ of the cells in the principal trigeminal nucleus ( $n=313$ ) have single-whisker receptive fields, whereas the rest of the population have receptive fields composed of multiple whiskers. The tonic or phasic properties of the re- sponses apparently bear no relation to the axonal projection patterns. Solid retrograde labeling of cells that project to the ventral posteromedial nucleus and intracellular staining revealed that single-whisker cells have small somata and narrow, barrelette-bounded dendritic trees. In contrast, multi-whisker neurons have large multipolar somata, expansive dendritic trees, and many respond antidromically to stimulation of the superior colliculus. Together, these results provide evidence for two main channels of vibrissal information: a single-whisker channel that links trigeminal barrelettes to their corresponding barreloids, and a multi-whisker channel that distributes principally in the posterior group and tectum.

Key words: vibrissa; barrel system; trigeminothalamic afferents; superior colliculus; posterior group nuclei; principal trigeminal nucleus
The rodent somatic sensory system is characterized by a prominent representation of the mystacial vibrissae. On each side of the rat snout, there are five horizontal rows of whiskers that form an orderly array of low-threshold mechanoreceptors. Each peripheral fiber innervating these mechanoreceptors responds to only one vibrissa and, centrally, the arrangement of the vibrissal pad is maintained in arrays of cellular aggregates referred to as barrelettes (brainstem), barreloids (thalamus), and barrels (cortex).

Brainstem nuclei that receive vibrissal primary afferents include the principal trigeminal nucleus (Pr5) and all subdivisions of the spinal trigeminal complex. Each of these (sub)nuclei contributes axons to the trigeminothalamic tract, but the main stream of ascending afferents arise from the Pr5 and interpolar division of the spinal complex. Many studies have investigated the responses of first-order afferents, thalamic, and cortical cells to vibrissae deflection (Armstrong-James, 1995; Diamond, 1995; Simons, 1995). In contrast, few physiological works have been devoted to the trigeminothalamic cells of the Pr5. The most comprehensive study (Shipley, 1974) described two broad classes of neurons: tonic units activated by a single whisker, and phasic units driven by single or multiple whiskers. Later studies mainly addressed anatomical issues, such as the way first-order afferents terminate in the Pr5 and how this nucleus innervates the thalamus and upper mesencephalon (Smith, 1975; Erzurumlu et al., 1980;

Received Jan. 19, 1999; revised March 17, 1999; accepted March 24, 1999.

This work was supported by a grant from the Medical Research Council of Canada.

Correspondence should be addressed to Martin Deschênes, Centre de Recherche Université Laval-Robert Giffard, Hôpital Robert-Giffard, 2601 de la Canardière, Québec G1J 2G3, Canada.

Copyright (C) 1999 Society for Neuroscience $\quad 0270-6474 / 99 / 195085-11 \$ 05.00 / 0$
Killackey and Erzurumlu, 1981; Huerta et al., 1983; Peschanski, 1984; Hayashi, 1985; Bruce et al., 1987; Jacquin et al., 1988; Jacquin and Rhoades, 1990; Chiaia et al., 1991; Bennett-Clarke et al., 1992; Williams et al., 1993). It was shown that, as a whole, the Pr5 projects massively to the ventral posteromedial nucleus (VPm) and, more sparsely, to the posterior group ( $\mathrm{Po}$ ) and superior colliculus. However, the way these projections are organized at a single cell level remains ill defined.

Labeling of a few whisker-sensitive Pr5 fibers at their entry in the thalamus revealed terminal arbors that are spatially restricted to the size of a barreloid (Williams et al., 1993). This is likely the case of the thicker axons, because bulk anterograde labeling also evidenced a Pr5 projection to Po. It was suggested, on the basis of double retrograde labeling, that $\sim 90 \%$ of the cells in the Pr5 project to VPm alone and $2 \%$ to both VPm and Po (Chiaia et al., 1991) and that most projections to the tectum and thalamus arise from separate populations of neurons (Bruce et al., 1987). However, the double retrograde labeling method may underestimate the number of branching axons if the injections do not precisely target the two sites that are innervated by the same fibers.

Intracellular labeling and retrograde tracer injections have disclosed two main types of projection neurons in the Pr5: small neurons with dendritic trees constrained to the dimension of a single barrelette, and large cells with more expansive dendritic trees (Jacquin et al., 1988; Jacquin and Rhoades, 1990; BennettClarke et al., 1992). The former category projects to V Pm, but it is not yet certain whether VPm is the only target of these neurons. The axonal projections of the large cells are unknown, although there is an evidence that some of them project to the superior colliculus (Bruce et al., 1987; Bennett-Clarke et al., 1992). In the present study, we sought to relate the response 
properties of whisker-sensitive cells in the $\operatorname{Pr} 5$ to their somatodendritic morphology and axonal projections.

\section{MATERIALS AND METHODS}

The present study was made in 40 adult rats (Sprague Dawley) in accordance with the federally prescribed and university animal care and use guidelines (Olfert et al., 1993).

Experiment 1. In a first series of experiments, 10 animals were anesthetized with ketamine $(75 \mathrm{mg} / \mathrm{kg})$ plus xylazine $(5 \mathrm{mg} / \mathrm{kg})$, and small deposits of biotinylated dextran amine (BDA) (molecular weight, 10,000; Molecular Probes, Eugene, OR) were made in the ventral division of the Pr5. Small-sized micropipettes (tip diameter, $4-5 \mu \mathrm{m}$ ) were filled with a solution of BDA $(2 \%)$ and K-acetate $(0.5 \mathrm{M})$ and lowered in the Pr5 according to the stereotaxic coordinates of the atlas of Paxinos and Watson (1986). After recording vibrissae-evoked multiunit activities to ascertain the correct placement of the micropipette, the tracer was ejected by iontophoresis (positive current pulses of $400-700 \mathrm{nA} ; 200$ msec duration; half duty cycle for $30 \mathrm{~min}$ ). This procedure was performed bilaterally, and animals were allowed to survive for $4 \mathrm{~d}$. They were perfused under deep urethane anesthesia $(1.4 \mathrm{gm} / \mathrm{kg})$ with a solution of $4 \%$ paraformaldehyde and $0.5 \%$ glutaraldehyde. Brains were cryoprotected in $30 \%$ sucrose overnight and cut horizontally at $75 \mu \mathrm{m}$ on a freezing microtome. Sections were serially collected in PBS and processed for cytochrome oxidase and BDA histochemistry according to previously described protocols (Wong-Riley, 1979; Horikawa and Armstrong, 1988). Darkly labeled trigeminothalamic axons were drawn with a camera lucida using $25 \times$ or $40 \times$ objectives.

Experiment 2. A second series of experiments $(n=18)$ were conducted under ketamine-xylazine anesthesia to determine the receptive field and response properties of single $\operatorname{Pr} 5$ neurons. Fine micropipettes $(\sim 1 \mu \mathrm{m})$ were pulled from thick-walled glass tubing, and the Pr5 was reached by passing through the cerebellum. Micropipettes were filled either with a solution of K-acetate $(3 \mathrm{M})$ or with a solution of K-acetate $(0.5 \mathrm{M})$ plus Neurobiotin (2\%; Vector Laboratories, Burlingame, CA). In some experiments, a pair of tungsten electrodes (tip diameter, $\sim 50 \mu \mathrm{m}$; spacing, $\sim 1 \mathrm{~mm}$ ) was placed in the deep layers of the contralateral superior colliculus to invade antidromically the Pr5 units that project to the tectum. Antidromic responses were identified by their fixed latency, ability to follow stimuli at $300 \mathrm{~Hz}$, and collision with orthodromic spikes triggered by the juxtacellular application of depolarizing currents.

For these experiments, vibrissae were clipped at $1 \mathrm{~cm}$ from the mystacial pad and colored to facilitate their identification. Under ketamine anesthesia, most Pr5 neurons were silent. Sometimes, neurons discharged spontaneously in association with isolated twitches or small tremors of the whiskers. Both motor activities and spontaneous discharges were abolished by an additional dose of anesthetics. When unit responses were seen to result from vibrissa deflection, manual stimulation was performed with a thin rigid rod under a dissecting microscope to provide a preliminary classification of the unit. First, it was determined whether a unit responded to a single or to multiple vibrissae. In the latter case, care was taken that the unit was not activated by stimulation of the intervibrissa fur or by deformation of the mystacial pad. Then, the unit was classified as tonic or phasic by deflecting the vibrissa in the direction that produced the most robust responses. This test was made by means of a three-axis, manually driven manipulator to avoid a false positive identification of tonic units that could result from the hand tremor of the experimentor. Units were classified as tonic if they fired throughout a 2-3 sec period of steady displacement of the corresponding whisker. A potential pitfall in these experiments is the possibility of recording from whisker-sensitive primary axons that travel just lateral to the Pr5 in the main trigeminal tract. In the very first experiments, this possibility was suspected because some descents yielded an anomalous number of axonal recordings characterized by monopolar positive spikes, an absence of response to juxtacellular current injections, and a lack of synaptic potentials or injury discharges after impalement. Intracellular labeling with Neurobiotin confirmed that such units were whisker-sensitive firstorder axons. These data were deleted from the database, and axonal recordings were disregarded in subsequent experiments.

Experiment 3. The aim of these experiments was to obtain a Golgi-like retrograde labeling of the Pr5 cells that project to the VPm. Large injections of BDA were made in two rats under ketamine-xylazine anesthesia. The tracer was ejected from large-sized micropipettes (tip diameter, $100 \mu \mathrm{m})$ filled with a solution of BDA (4\%) and K-acetate $(0.2$ $\mathrm{M})$. Injections were made bilaterally by passing positive current pulses of
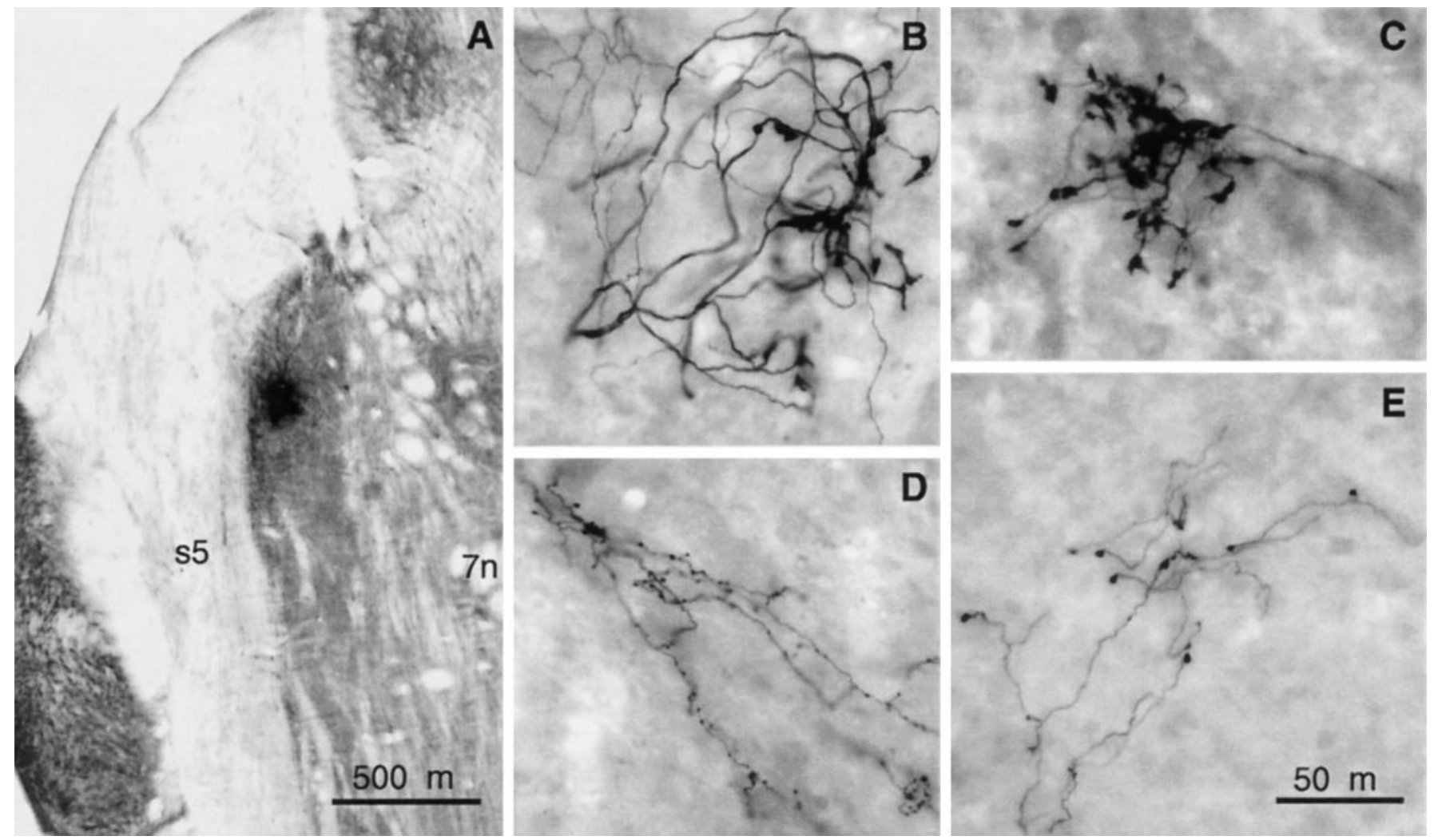

Figure 1. Photomicrographs of an injection site in the $\operatorname{Pr} 5(A)$ and of terminal fields in VPm $(B, C)$, superior colliculus $(D)$, and Po $(E)$. All pictures were taken from horizontal sections. Scale bar in $E$ also applies to $B-D$. $7 n$, Seventh nerve tract; $s 5$, spinal trigeminal tract. 

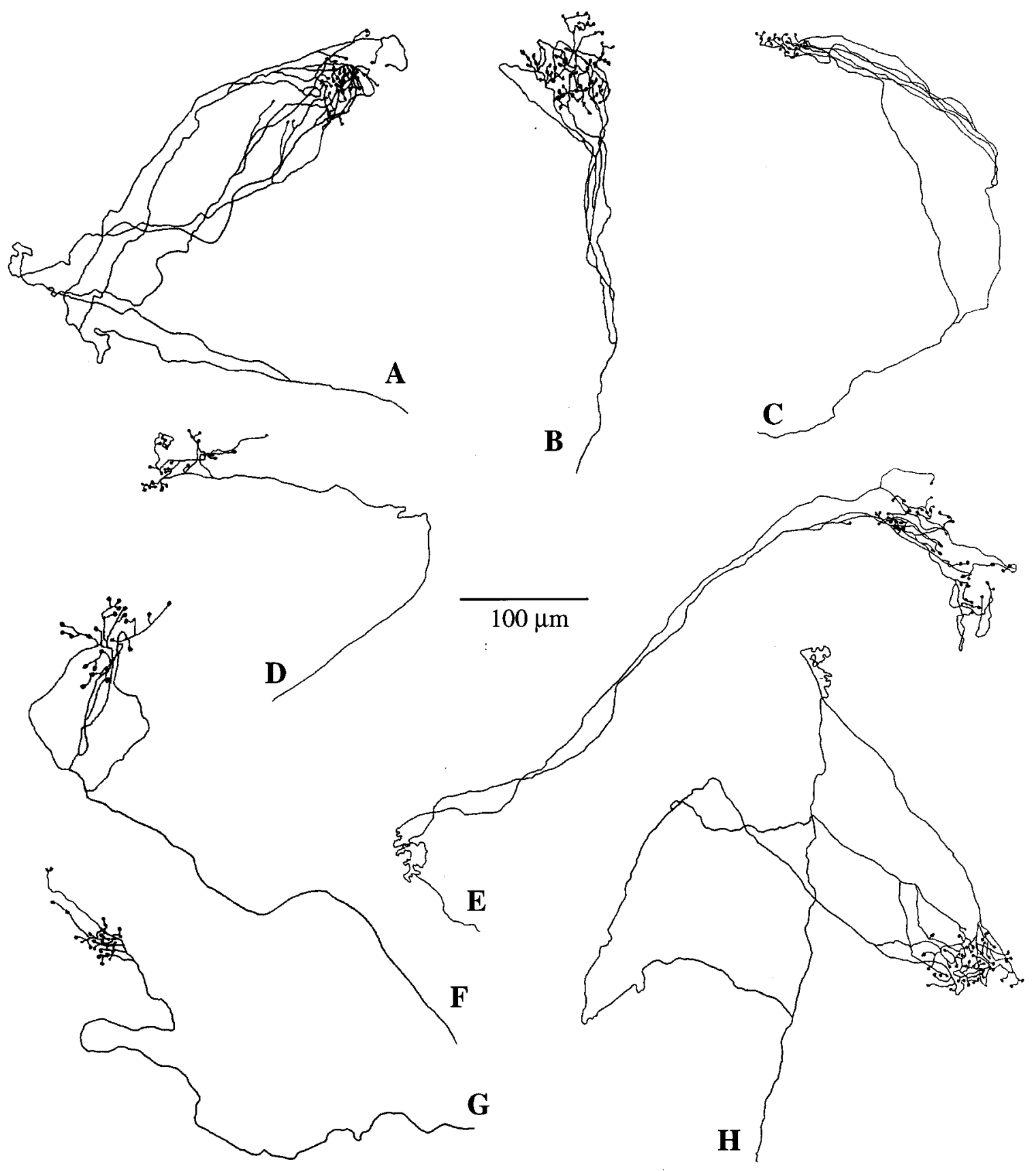

Figure 2. Terminal fields of Pr5 axons that innervate a single barreloid in the rat VPm. All reconstructions were made from horizontal sections.

$4 \mu \mathrm{A}$ ( $7 \mathrm{sec}$ on-off) for $30 \mathrm{~min}$. After a survival period of $4 \mathrm{~d}$, animals were perfused, and the tissue was processed for BDA histochemistry.

Experiment 4. In these experiments, whisker-sensitive Pr5 cells were labeled intracellularly after identification of their receptive field. Ten rats were anesthetized with either ketamine-xylazine or urethane $(1.4 \mathrm{gm} /$ $\mathrm{kg}$ ), and vibrissae stimulation was performed as described above. After removing the cerebellum, the Pr5 was reached by lowering the micropipette obliquely at an angle of $30^{\circ}$. A fork made of two fine tungsten rods (diameter, $100 \mu \mathrm{m}$; spacing, $1 \mathrm{~mm}$ ) was inserted into the pons to minimize respiratory and cardiac pulsations. Despite the remaining pulsations, this method permitted short-duration intracellular recording periods (4-5 min) sufficient to characterize the functional properties of the cells and label them with Neurobiotin. Micropipettes contained 2\% Neurobiotin in $0.5 \mathrm{~m} \mathrm{~K}$-acetate, and intracellular labeling was achieved by injecting positive current pulses of 1-3 nA for 3-5 min. At the end of the experiments, animals were perfused, and the tissue was processed for
Neurobiotin histochemistry according to a previously described protocol (Horikawa and Armstrong, 1988).

\section{RESULTS}

\section{The axonal projections of Pr5 neurons}

The morphological database consists of 107 trigeminothalamic axons that were traced from 16 injection sites distributed in the ventral part of the Pr5 (Fig. 1). Each injection resulted in the solid dark labeling of 2-10 trigeminothalamic fibers. The arborizations of 32 axons were completely drawn, and the others were partially reconstructed to verify whether they display branching patterns similar to those of the fully reconstructed fibers.

On the basis of the location of terminal fields in the thalamus, 


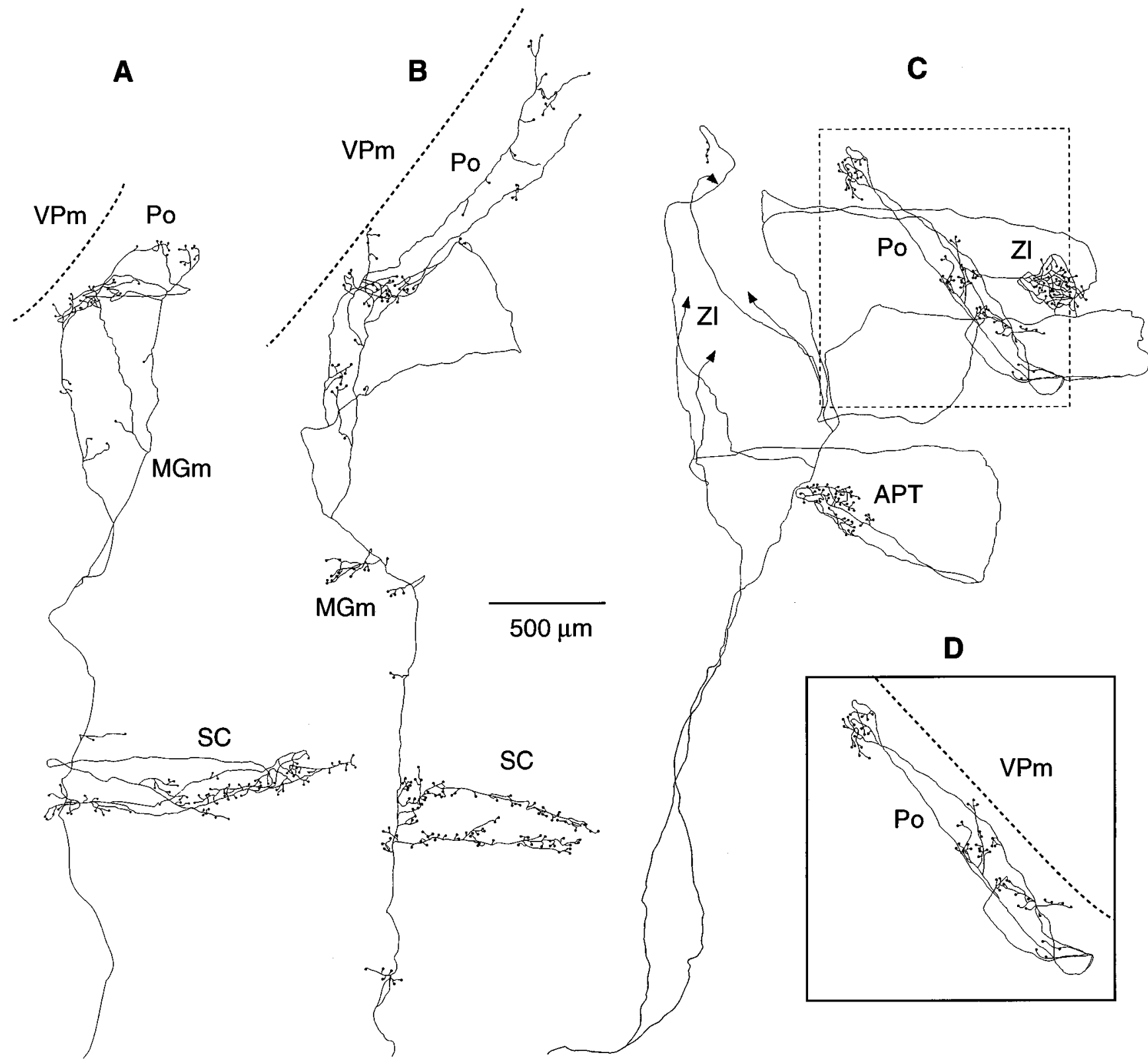

Figure 3. Axonal arborizations of three Pr5 cells that project to Po and upper brainstem. Axons in $A$ and $B$ innervate the superior colliculus ( $S C$ ), MGm, and Po. Axon in $C$ innervates the anterior pretectal nucleus $(A P T)$, zona incerta $(Z I)$, and Po. Framed drawing in $D$ shows the terminal field in Po for the axon illustrated in $C$. Note the distribution of boutons at the border of VPm. All reconstructions were made from horizontal sections.

one can distinguish two main groups of Pr5 axons: those that project to a single barreloid (type 1), and those that project to both Po and other mesencephalic or diencephalic regions (type 2). These two types of fibers were labeled together after an injection, thus showing that the cells of origin are not spatially segregated within the Pr5.

Type 1 axons, which account for $68 \%$ of the sample, innervate only the contralateral VPm. These axons cross the midline at the level of the rostral pons and travel without branching in the medial lemniscus. They ascend through the lateral thalamus, where they generate a cluster of branches that finally clump together to form a small terminal field restricted to the dimension of a single barreloid (Fig. 2). Terminal fields are grossly spherical (diameter, 60-100 $\mu \mathrm{m}$ ) and contain 25-60 large terminations (Fig. 1B,C).
Like the fibers of the first group, type 2 axons cross the midline in the pons, travel in the medial lemniscus and, at approximately the level of the red nucleus, give off a thick ascending branch that heads toward the tectum (Fig. 3). These fibers, which represent $23 \%$ of the ascending Pr5 afferents, project to Po. Terminal fields in Po are sparse (Fig. 1E), contain a small number of large terminations $(\sim 4 \mu \mathrm{m})$, and are mainly concentrated at the border of the VPm. As a rule, these axons also innervate the intermediate white and deep gray layers of the superior colliculus or, less frequently, the anterior pretectal nucleus. In both structures, they form dense mediolaterally oriented bands of terminations (Fig. $1 D)$. Additional terminal sites for these axons include the ventral part of the zona incerta and/or the medial part of the medial geniculate nucleus (MGm).

Aside from these two main classes of axons, a minority of fibers 
A

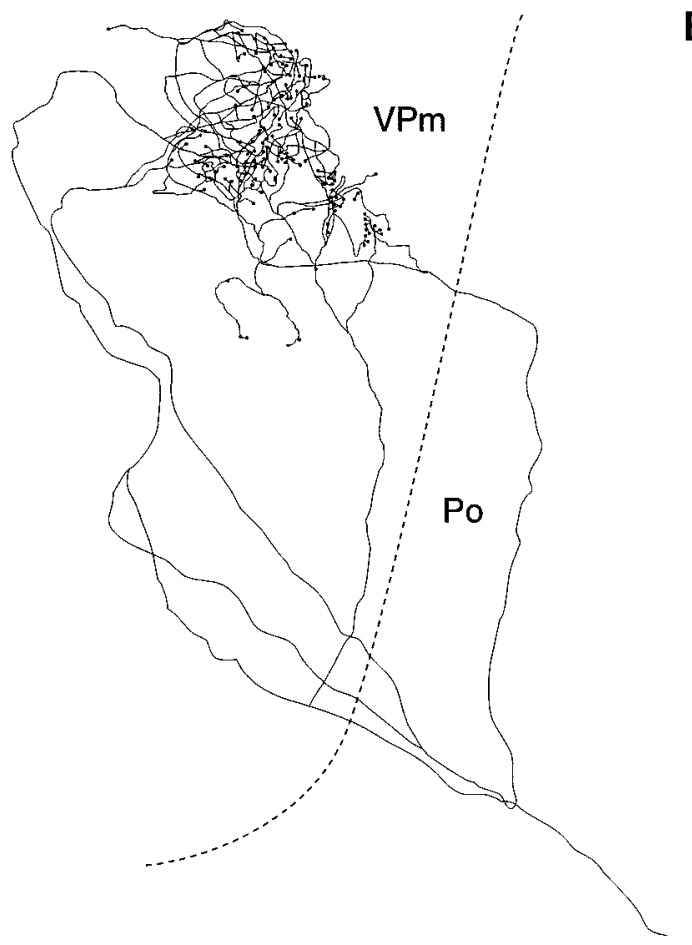

B

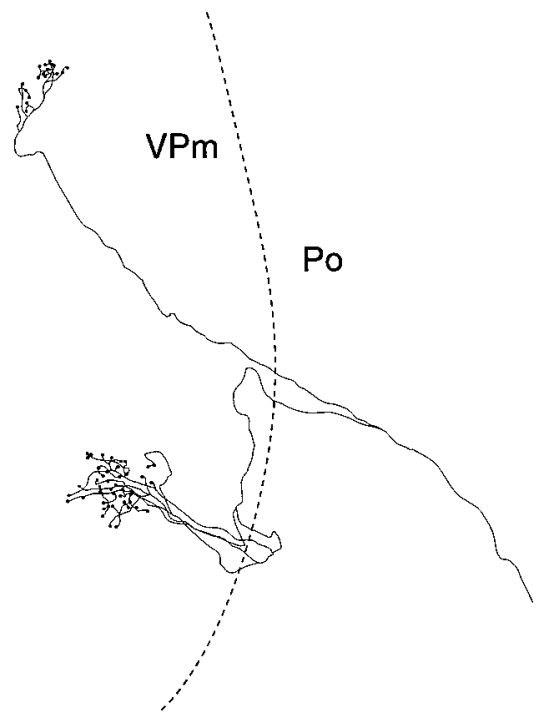

$200 \mu \mathrm{m}$
Figure 4. Axonal arborizations of two Pr5 cells in the rat VPm. The terminal field in $A$ is larger than the average size of a single barreloid, and the axon in $B$ forms two separate puffs of terminations. Reconstructions were made from horizontal sections.
(5\%) form two clusters of large terminations in different regions of the VPm or generate, in the dorsal VPm, a single terminal field that is more expansive than the average dimension of a barreloid (Fig. 4).

The last category of fibers, which represent $4 \%$ of the total population of Pr5 afferents, project to both VPm and Po and also innervate the tectum and the MGm (Fig. 5). The terminal fields in VPm stretch rostrocaudally in the dorsal part of the barreloids and contain a moderate number of large terminations. Like the type 2 fibers, these axons form a loose plexus in Po with few scattered boutons.

\section{Response properties of Pr5 neurons}

A second series of experiments was aimed at determining whether whisker-sensitive Pr5 neurons exhibit functional properties that may be related to their patterns of projection. We thus studied the response properties of a large sample of Pr5 cells and used the antidromic invasion technique to identify cells that project to the tectum.

The physiological database consists of 313 units that responded exclusively to whisker displacement. Two hundred eight units $(67 \%)$ had a receptive field confined to a single vibrissa. Among these cells, tonic and phasic units account, respectively, for 38 and $62 \%$ of the population. Figure 6 shows a series of histograms depicting the number of single-whisker units that respond either tonically or phasically to each of the whiskers of the mystacial pad. Although the most rostral vibrissae might be underrepresented in the sample, no clear trend toward tonicity or phasicity seems to characterize units with respect to the rostrocaudal position of their corresponding whisker on the pad. A majority of these units were also found differentially sensitive to the direction of displacement. However, this test was conducted too crudely, with a hand-held probe, to relate this feature to the other response properties of the cells.

The remaining units (33\% of the sample) had receptive fields composed of 2-14 contiguous vibrissae, with one of the vibrissae eliciting a more robust response. The set of whiskers that activated any of these units was preferentially distributed rostrocaudally along the pad. It was estimated, by computing the ratio between the number of effective vibrissae in the longest arc versus the longest row, that the average receptive field of a multi-whisker unit was three arcs long and two rows wide $(n=60$ receptive fields). Most multi-whisker units (99 of 105) responded phasically to vibrissae displacement and exhibited directional selectivity.

Antidromic activation of the Pr5 units was attempted from the superior colliculus rather than from the thalamus, because thalamic stimulation could not be used to discriminate between axons that project to VPm alone from those that project to the neighboring Po. All cells that could be activated antidromically from the tectum were multi-whisker units $(n=38$; latency, $0.9 \pm$ $0.1 \mathrm{msec})$. In contrast, none of the single-whisker units tested $(n=$ 112) could be backfired from the superior colliculus.

\section{Morphological identification of Pr5 cells}

Golgi-like labeling of Pr5 cells was obtained from two of the four injections made in the VPm. Figure 7 shows the extent of cellular backfilling and the shape of some VPm-projecting neurons. The vast majority of the cells have small oval somata $(8 \times 12 \mu \mathrm{m})$, with dendrites showing a high degree of streaming along the rostrocaudal axis of the nucleus. A minority of cells with larger cell bodies $(\sim 25 \mu \mathrm{m})$ are present and also harbor highly polarized dendritic trees.

Eleven whisker-sensitive cells were individually labeled with Neurobiotin after the characterization of their sensory responses. This sample includes six single-whisker and five multi-whisker units, which were all located within the limits of the Pr5. Singlewhisker cells have small somata and narrow dendritic trees strongly polarized along the rostrocaudal axis of the Pr5 (Figs. $8 B, 9 A)$. Typical dendritic fields are 80-100 $\mu \mathrm{m}$ wide and 400$700 \mu \mathrm{m}$ long. In contrast, the multi-whisker units have large multipolar cell bodies and have a more expansive dendritic tree that span across multiple Pr5 barrelettes (Figs. $8 A, 9 B$ ). Dendritic 


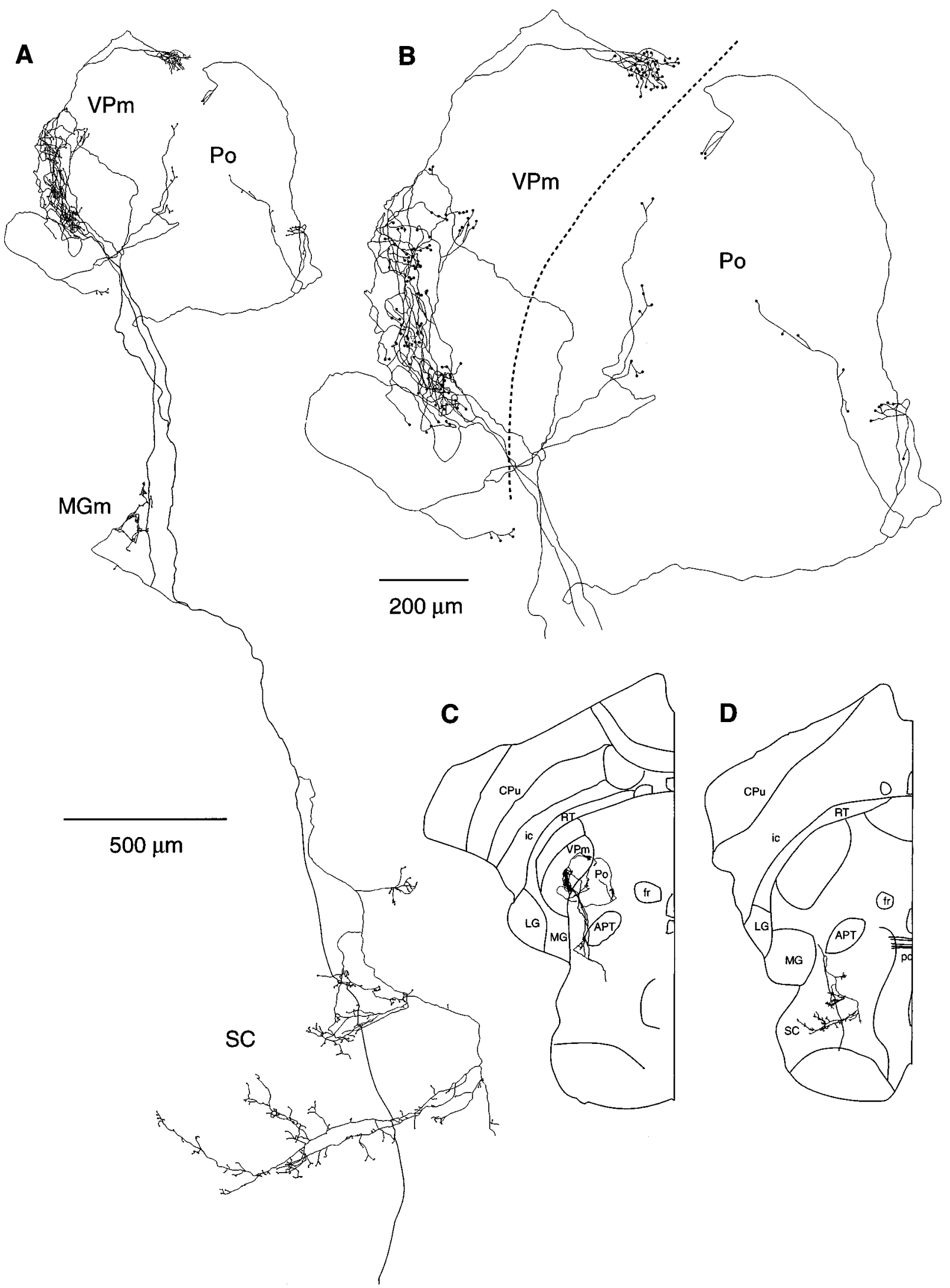

Figure 5. Axonal projections of a Pr5 cell that projects to both VPm and Po. $B$ shows the distribution of terminations in the thalamus. $C$ and $D$ show the location of terminal fields on horizontal sections at low magnification. $A P T$, Anterior pretectal nucleus; $C P u$, caudate/putamen; $f r$, fasciculus retroflexus; $i c$, internal capsule; $L G$, lateral geniculate nucleus; $M G$, medial geniculate nucleus; $p c$, posterior commissure; $R T$, reticular thalamic nucleus; $S C$, superior colliculus. 
A

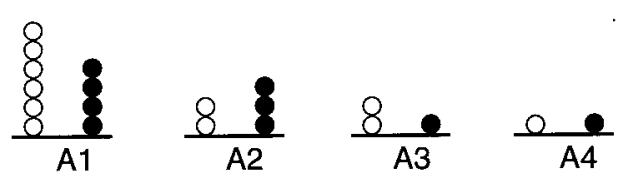

\section{$\frac{8 \text { : }}{\alpha}$}

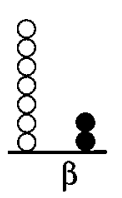

8!
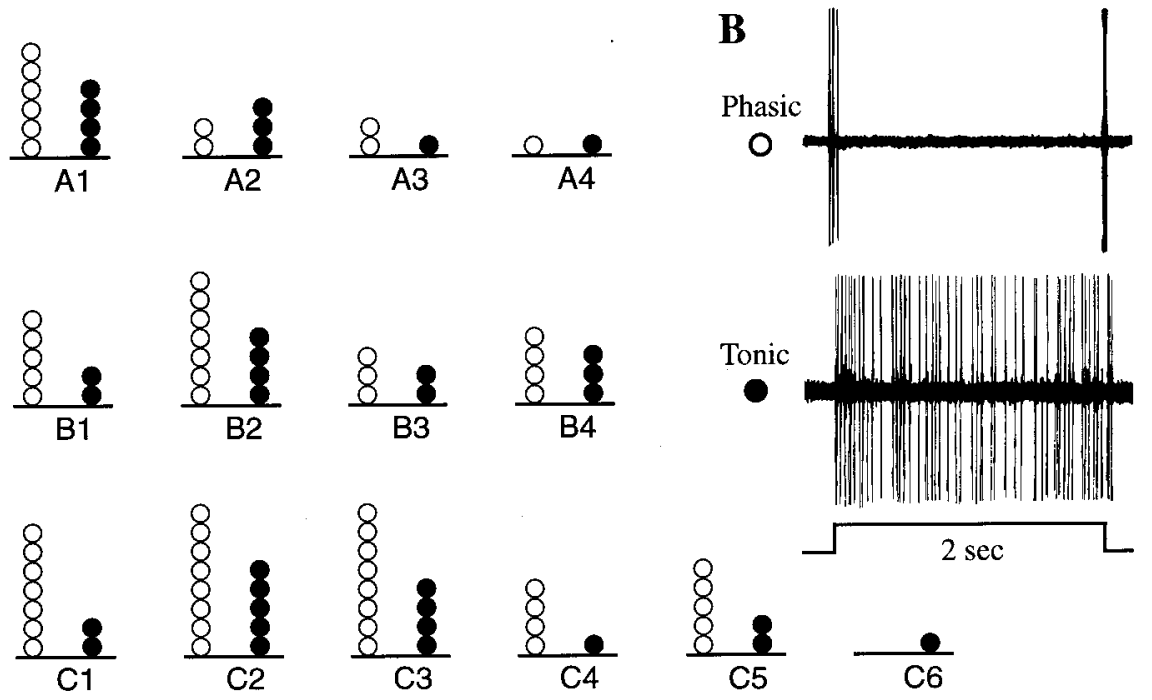
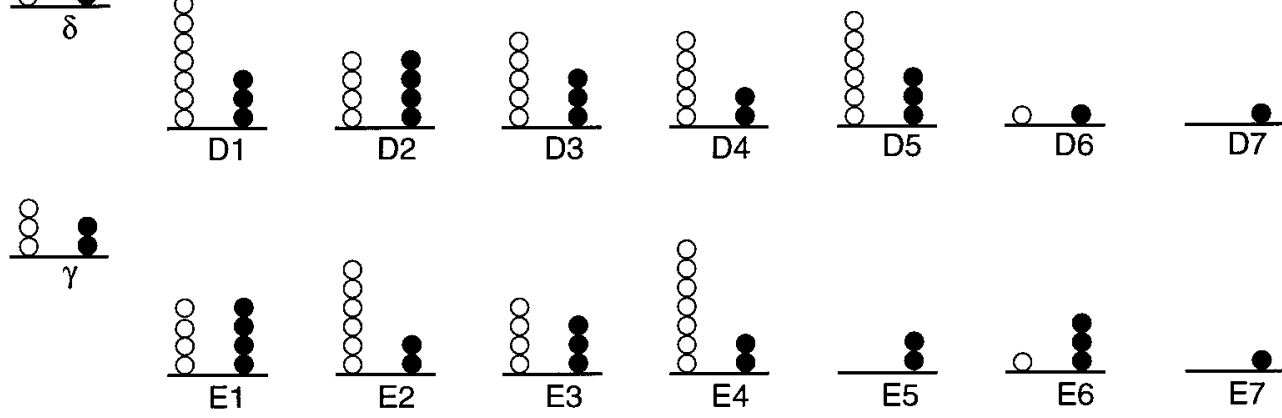

trees do not exhibit any fixed orientation but seem to conform with the topographic representation of the vibrissae to which they respond.

\section{DISCUSSION}

The projection sites of the Pr5 in rats have already been described in studies using the techniques of neuronal degeneration or axonal transport. In addition to the VPm, the Pr5 was found to project to Po (Smith, 1975; Chiaia et al., 1991; Williams et al., 1993), MGm (Peschanski, 1984), superior colliculus (Erzurumlu et al., 1980; Killackey and Erzurumlu, 1981; Huerta et al., 1983; Bruce et al., 1987), the ventral part of the zona incerta (Smith, 1975; Roger and Cadesseau, 1983; Peschanski, 1984; ShammahLagnado et al., 1985; Nicolelis et al., 1992), and the anterior pretectal nucleus (Yoshida et al., 1992). The same projection sites were found in the present study, which shows how these multiple projections are generated at a single-cell level. Two main classes of trigeminothalamic cells were identified: cells with a restricted terminal field in VPm, and cells that project to Po, as well as to other mesencephalic and diencephalic targets. Figure 10 shows a summary diagram of these projections.

\section{Methodological comments}

Three methodological aspects of the present study require comments.

(1) The most direct way to study the relationship between the functional properties and the axonal projection patterns of Pr5 cells would have been to record their responses to whisker stimulation and label them with a tracer. This approach, however,

proved impractical because brain pulsations impeded the stability required by the intracellular or juxtacellular labeling methods. We thus relied on a correlative approach, which consists in analyzing large samples of morphological and physiological data. This approach has limitations, especially when attempts are made to relate data among small subgroups of neurons with different anatomical and physiological features. It seems likely that the diversity of projection patterns of the multi-whisker cells, for instance, may be related to different response properties. A more rigorous analysis of these properties together with cell labeling would be required to settle this issue. The same qualification applies to the morphology of single-whisker cells, which, despite similar dendritic domains, might display morphological differences related to their tonic or phasic properties, and/or the location of their corresponding whisker on the mystacial pad.

(2) Although the injections of BDA were made in the ventral whisker-responsive region of the Pr5, it is not excluded that some labeled fibers might have receptive fields on neighbor regions of the mystacial pad (i.e., guard hairs, intervibrissa fur). Our data show, however, a very tight correspondence between the proportion of fibers that project to a single barreloid and the proportion of single-whisker sensitive cells in the $\operatorname{Pr} 5$ (68 vs 67\%). It thus seems unlikely that the anatomical database was much contaminated by nonwhisker related axons.

(3) The reconstruction of Pr5 axons was made from horizontal sections processed for cytochrome oxidase. Although background staining clearly delineates the boundaries of the Pr5 and VPm nuclei, this plane of sectioning does not allow the visualization of 


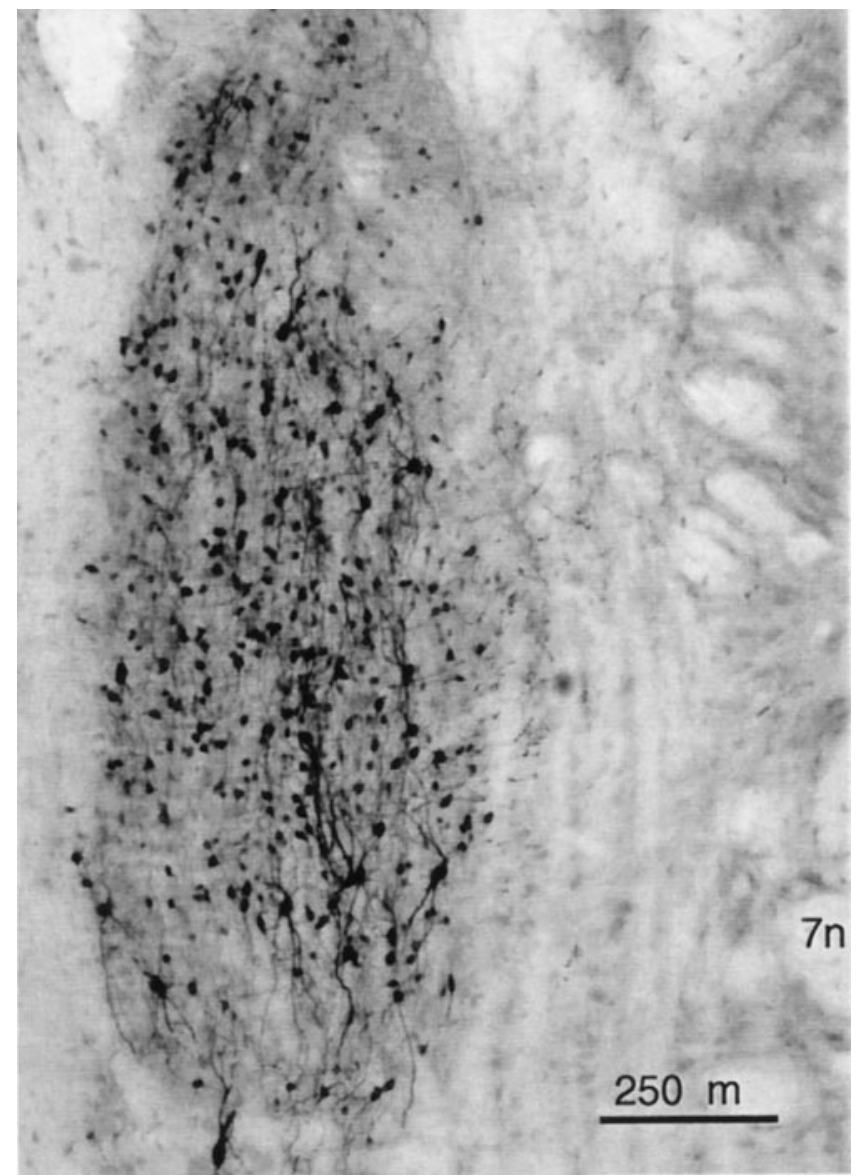

Figure 7. Solid retrograde labeling of Pr5 cells after an injection of BDA in the VPm. Note the polarization of dendritic trees along the rostrocaudal axis. $7 n$, Seventh nerve tract. the individual whisker-related modules, such as the barrelettes and barreloids. We thus relied on previous descriptions of these modules in rats to refer to their architecture and somatotopic organization (Henderson and Jacquin, 1995; Land et al., 1995).

\section{Single- versus multi-whisker channels}

Previous studies of whisker-sensitive cells in the rat Pr5 reported a majority of units with receptive fields confined to a single vibrissa and a minority that responded to the displacement of multiple whiskers. The former group accounted for $68 \%$ of the cells in Shipley's study (1974), which is close to the percentage (67\%) found in the current work. The remaining percentage of multi-whisker cells $(\sim 33 \%)$, however, is somewhat higher than the proportion reported in other studies (20\% in Jacquin et al., 1988; 23\% in Doherty et al., 1993). This difference may be attributable to the use of different levels of anesthesia or to different cell samples that include either some first-order trigeminal axons or some neurons from the oralis division of the spinal trigeminal nucleus.

There is now strong evidence that single-whisker cells in the Pr5 nucleus project only to their corresponding barreloid. Our current study shows a remarkably tight correspondence between the proportion of Pr5 axons that project to a single barreloid $(68 \%)$ and the proportion of cells with single-whisker receptive fields $(67 \%)$. These correlative results extend and confirm those of Williams et al. (1993), who directly demonstrated, in a small sample of intracellularly stained fibers, that single-whisker Pr5 axons project to a single barreloid.

Although retrograde and anterograde transport studies provided evidence of a Pr5 projection to Po, cells of origin of this projection and their patterns of axonal distribution remained ill defined. In double retrograde labeling experiments, it was estimated that $\sim 90 \%$ of the cells in the Pr5 project to VPm alone and $2 \%$ to both VPm and Po (Chiaia et al., 1991). This leaves $\sim 8 \%$ of fibers that innervate Po alone, a proportion approximately three times smaller than that observed in our anatomical survey $(23 \%)$. This contrasting result likely reflects the fact that the
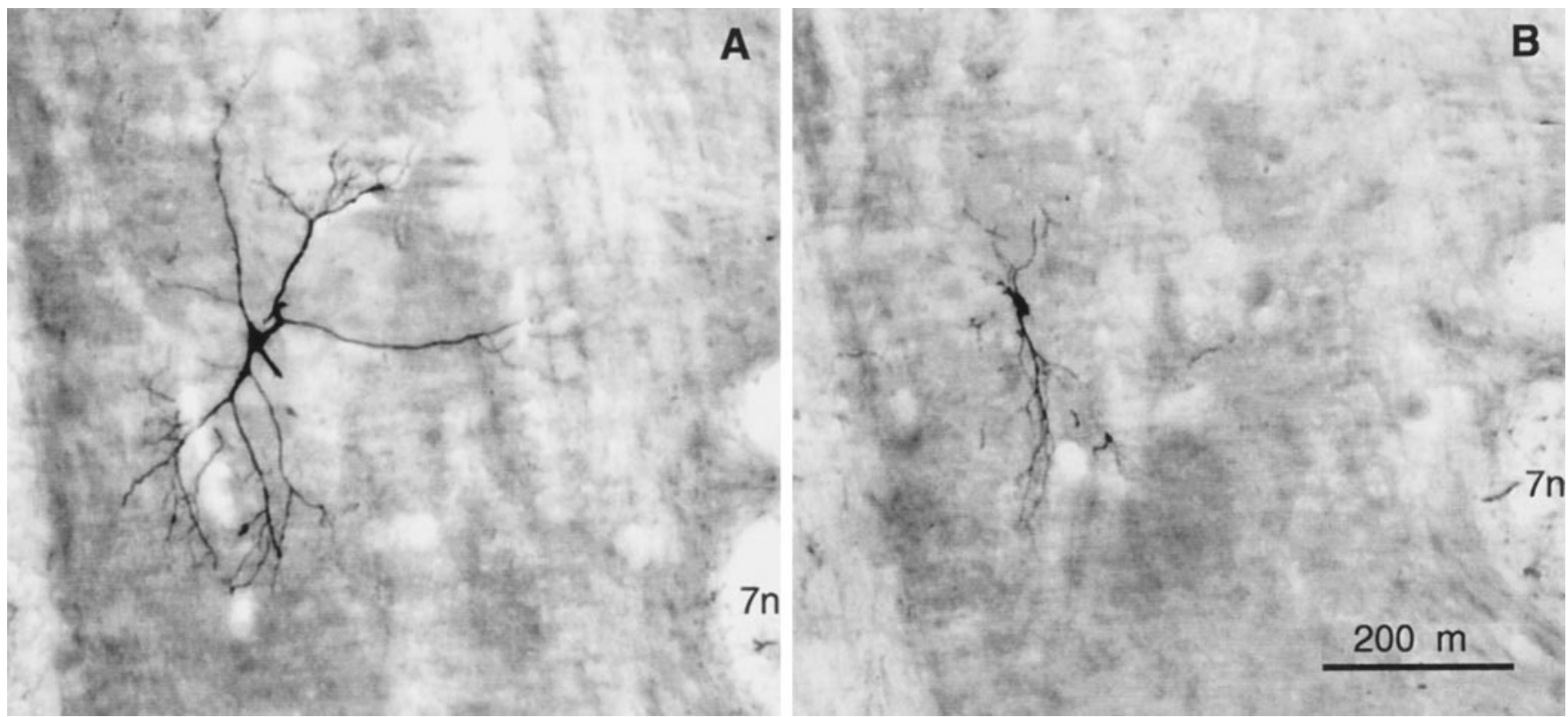

Figure 8. Photomicrographs of Pr5 cells labeled intracellularly with Neurobiotin. The cell in $A$ was backfired from the superior colliculus and responded to the displacement of whiskers D3, D4, and D5. B, Single-whisker phasic cell sensitive to whisker C4. 


\section{A}

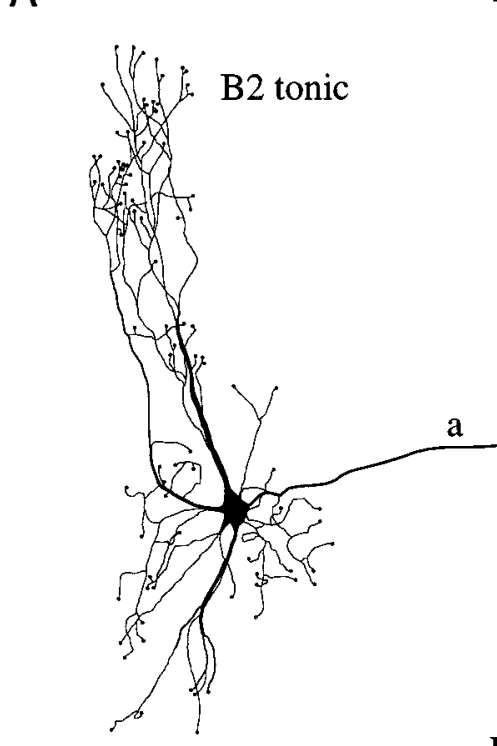

E

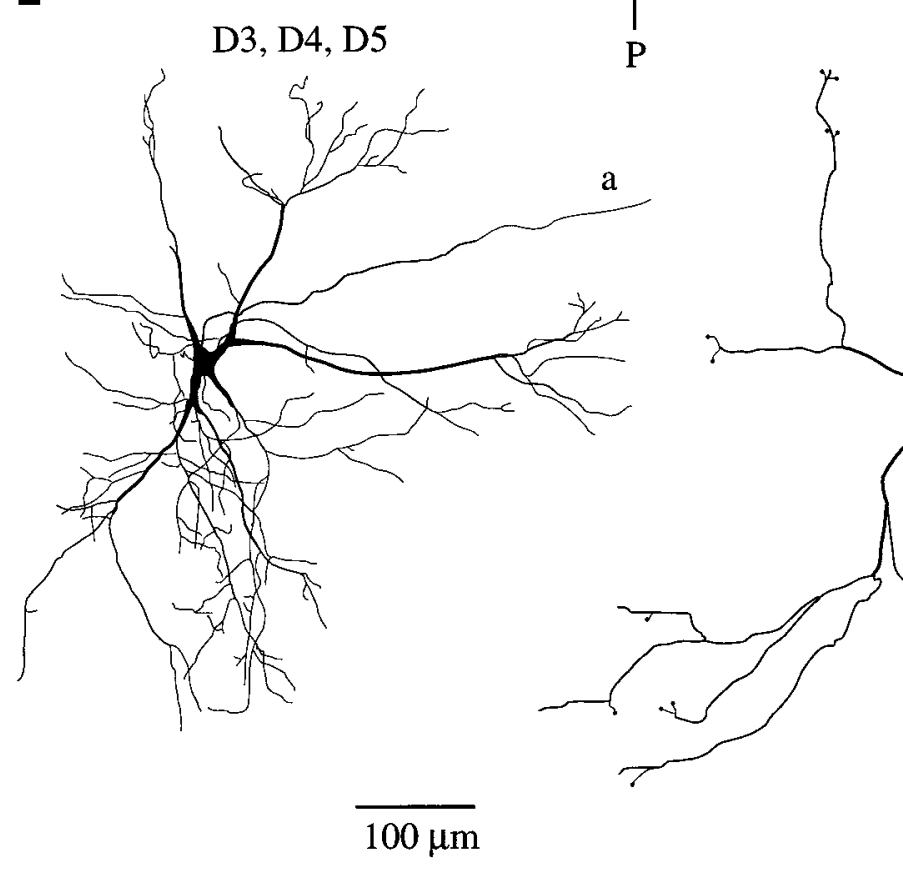

M

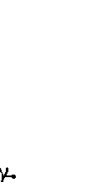

C
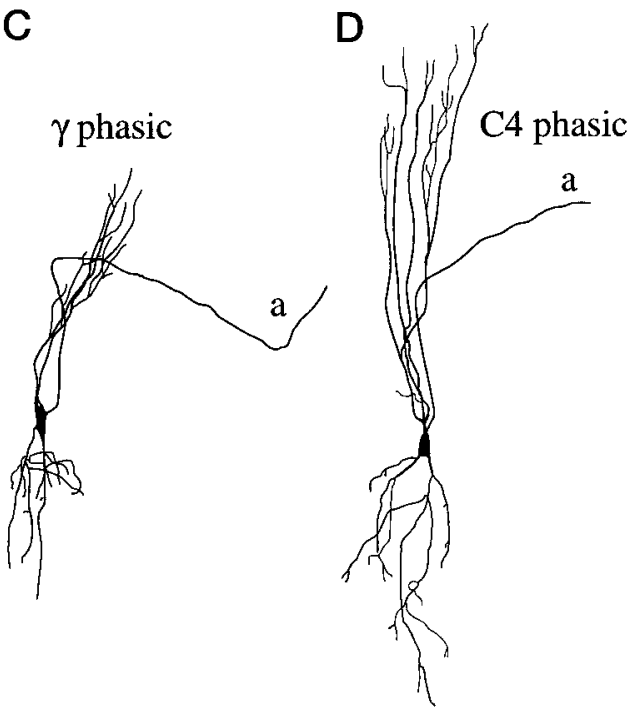

B

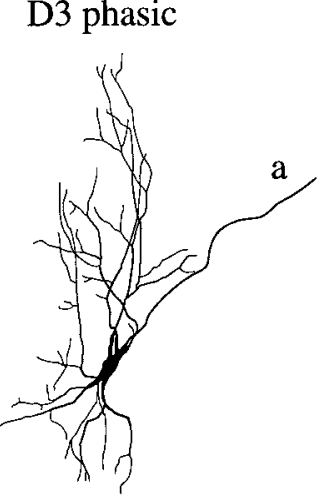

(1)
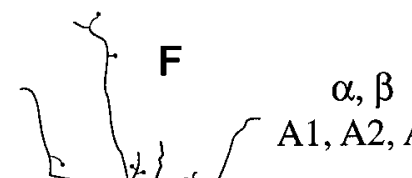

$\alpha, \beta$

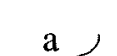




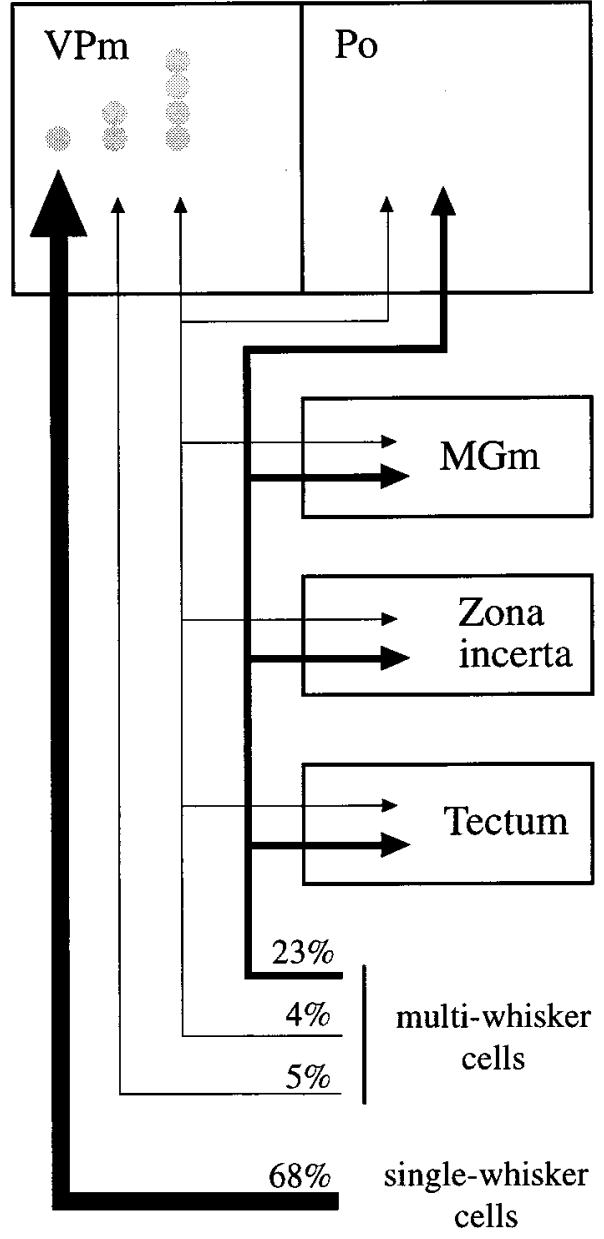

Figure 10. Schema of the ascending projections of whisker-sensitive cells of the Pr5. Percentages were computed from a population of 107 axons reconstructed after anterograde staining with BDA.

in Bruce et al., 1987). All studies agree, however, on the fact that this projection arises from the large multipolar cells that were found to respond to the deflection of multiple whiskers (Bruce et al., 1987; Bennett-Clarke et al., 1992; Henderson and Jacquin, 1995).

\section{Tonic and phasic units}

Phasic and tonic units represent, respectively, 62 and $38 \%$ of the Pr5 cells with single-whisker receptive fields. Both types of units are likely represented in the population of axons that were traced to VPm alone. However, no obvious morphological feature permits us to distinguish subgroups among these axons. Functional subdivisions may be present within the barreloids; their dorsomedial parts were reported to be more reactive for cytochrome oxidase (Land et al., 1995), and V Pm neurons that exhibit rapidly adapting responses are more commonly recorded in this dorsal region (Ito, 1988). It is thus possible that tonic and phasic afferents have similar morphologies but terminate in different regions of barreloids. Whether a spatial segregation of this sort exists in the rat VPm remains an open issue.

\section{Place coding and parity in the vibrissa sensory system}

Studies of primary vibrissa afferents in the rat agree on a number of general points. Trigeminal ganglion cells have single-whisker receptive fields, are either slowly or rapidly adapting, and exhibit sensitivity to the direction of movements (Zucher and Welker, 1969; Gibson and Welker, 1983a,b; Lichtenstein et al., 1990). The central projections of slowly adapting axons are undistinguishable from those of rapidly adapting fibers. In the Pr5, both form several terminal clusters that are spatially aligned within the barrelette corresponding to their single-whisker receptive field (Henderson and Jacquin, 1995). Place specificity is maintained in the Pr5-VPm projections by single-whisker sensitive cells that have barrelette-bounded dendritic trees and project to a single barreloid. Likewise, place coding is conserved in the reciprocal connections between barreloids and their corresponding barrel columns in the cortex (Land et al., 1995). There is, however, a remarkable difference in the way Pr5 and corticothalamic axons innervate a thalamic barreloid. Whereas single Pr5 afferents target only part of a barreloid, single corticothalamic axons ramify throughout its full extent (Bourassa et al., 1995). For the corticothalamic fibers to innervate the full extent of a barreloid, some whisker-specific recognition sites might be induced in thalamic neurons that receive Pr5 inputs from the same vibrissa. This possibility would be in line with the rule of parity, which was recently proposed as a new organization principle of the connections between the cortex and thalamus (Deschênes et al., 1998).

\section{REFERENCES}

Armstrong-James M (1995) The nature and plasticity of sensory processing within adult rat barrel cortex. In: Cerebral cortex, Vol 12, The barrel cortex of rodents (Jones EG, Diamond IT, eds), pp 333-373. New York: Plenum.

Bennett-Clarke CA, Chiaia NL, Jacquin MF, Rhoades RW (1992) Parvalbumin and calbindin immunocytochemistry reveal functionally distinct cell groups and vibrissa-related patterns in the trigeminal brainstem complex of the adult rat. J Comp Neurol 320:323-338.

Bourassa J, Pinault D, Deschênes M (1995) Corticothalamic projections from the cortical barrel field in rats: a single fiber study using biocytin as an anterograde tracer. Eur J Neurosci 7:19-30.

Bruce LL, McHaffie JG, Stein BE (1987) The organization of trigeminotectal and trigeminothalamic neurons in rodents: a double-labeling study with fluorescent dyes. J Comp Neurol 262:315-330.

Chiaia NL, Rhoades RW, Bennett-Clarke CA, Fish SE, Killackey HP (1991) Thalamic processing of vibrissal information in the rat. I. Afferent input to the medial ventral posterior and posterior nuclei. J Comp Neurol 314:201-216.

Deschênes M, Veinante P, Zhang Z-W (1998) The organization of corticothalamic pathways: reciprocity versus parity. Brain Res Rev 28:286-308.

Diamond ME (1995) Somatosensory thalamus of the rat. In: Cerebral cortex, Vol 12, The barrel cortex of rodents (Jones EG, Diamond IT, eds), pp 189-219. New York: Plenum.

Doherty DW, Jacquin MF, Killackey HP (1993) Quantitative analysis of receptive field properties in the rat nucleus principalis. Soc Neurosci Abstr 19:327.

Erzurumlu RS, Bates CA, Killackey HP (1980) Differential organization of thalamic projection cells in the brain stem trigeminal complex of the rat. Brain Res 198:427-433.

Gibson JM, Welker WI (1983a) Quantitative studies of stimulus coding in first-order vibrissa afferents of rats. I. Receptive field properties and threshold distributions. Somatosens Res 1:51-67.

Gibson JM, Welker WI (1983b) Quantitative studies of stimulus coding in first-order vibrissa afferents of rats. II. Adaptation and coding of stimulus parameters. Somatosens Res 1:95-117.

Hayashi H (1985) Morphology of central terminations of intra-axonally stained large myelinated primary afferent fibers from facial skin in the rat. J Comp Neurol 237:195-215.

Henderson TA, Jacquin MF (1995) What makes subcortical barrels? In: Cerebral cortex, Vol 12, The barrel cortex of rodents (Jones EG, Diamond IT, eds), pp 123-187. New York: Plenum. 
Horikawa K, Armstrong WE (1988) A versatile means of intracellular labeling: injection of biocytin and its detection with avidin conjugates. J Neurosci Methods 25:1-11.

Huerta MF, Frankfurter A, Harting JK (1983) Studies of the principal sensory and spinal trigeminal nuclei of the rat: projections to the superior colliculus, inferior olive and cerebellum. J Comp Neurol 220:147-167.

Ito M (1988) Response properties and topography of vibrissa-sensitive VPM neurons in the rat. J Neurophysiol 60:1181-1197.

Jacquin MF, Rhoades RW (1990) Structure and response properties of cells in the trigeminal subnucleus oralis. Somatosens Mot Res 7:265-288.

Jacquin MF, Golden JP, Panneton WM (1988) Structure and function of barrel precursor cells in trigeminal nucleus principalis. Dev Brain Res 43:309-314.

Killackey HP, Erzurumlu RS (1981) Trigeminal projections to the superior colliculus of the rat. J Comp Neurol 201:221-242.

Land PW, Buffer SA, Yaskoski JD (1995) Barreloids in adult rat thalamus: three-dimensional architecture and relationship to somatosensory cortical barrels. J Comp Neurol 355:573-588.

Lichtenstein SH, Carvell CA, Simons DJ (1990) Responses of rat trigeminal ganglion neurons to movements of vibrissae in different directions. Somatosens Mot Res 7:47-65.

Nicolelis MAL, Chapin JK, Lin RCS (1992) Somatotopic maps within the zona incerta relay parallel GABAergic somatosensory pathways to the neocortex, superior colliculus and brainstem. Brain Res 577:134-141.

Olfert ED, Cross BM, McWilliams AA (1993) Guide to the care and use of experimental animals. Canadian Council on Animal Care. Bradda, Ontario, Canada.
Paxinos G, Watson C (1986) The rat brain in stereotaxic coordinates, Ed 2. Sydney: Academic.

Peschanski M (1984) Trigeminal afferents to the diencephalon in the rat. Neuroscience 12:465-487.

Roger M, Cadesseau J (1983) Afferents to the zona incerta in the rat: a combined retrograde and anterograde study. J Comp Neurol 241:480-492.

Shammah-Lagnado SJ, Negrao N, Ricardo JA (1985) Afferent connections of the zona incerta: a horseradish peroxidase study in the rat. Neuroscience 15:109-134.

Shipley MT (1974) Response characteristics of single units in the rat's trigeminal nuclei to vibrissa displacements. J Neurophysiol 37:73-90.

Simons DJ (1995) Neuronal integration in the somatosensory whisker/ barrel cortex. In: Cerebral cortex, Vol 12, The barrel cortex of rodents (Jones EG, Diamond IT, eds), pp 263-297. New York: Plenum.

Smith RL (1975) The ascending fiber projections from the principal sensory trigeminal nucleus in the rat. J Comp Neurol 148:423-446.

Williams MN, Zahm DS, Jacquin MF (1993) Differential foci and synaptic organization of the principal and spinal projections to the thalamus in rats. Eur J Neurosci 6:429-453.

Wong-Riley MTT (1979) Changes in the visual system of monocularly sutured or enucleated cats demonstrable with cytochrome oxidase histochemistry. Brain Res 171:11-28.

Yoshida A, Sessle BJ, Dostrovski JO, Chiang CY (1992) Trigeminal and dorsal column nuclei projections to the anterior pretectal nucleus in the rat. Brain Res 590:81-94.

Zucker E, Welker WL (1969) Coding of somatic sensory input by vibrissae neurons in the rat's trigeminal ganglion. Brain Res $12: 138-156$ 